\title{
Habilidades gerenciales, visión del egresado Tomasino-VUAD
}

Management skills, vision of the graduate Tom a sino-VuAD

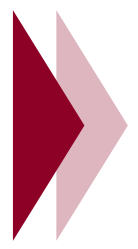

Martha Cecilia Pachón

Doctorando en Proyectos, Funiber; magister en Mercadeo, Universidad Externado de Colombia; especialista en Formulación y Evaluación Social y Económica de Proyectos, Universidad Católica de Colombia; administradora de empresas comerciales, Universidad Colegio Mayor de Cundinamarca. Docente Universidad Santo Tomás a distancia pregrado y postgrado.

E-mail: marthapachon@ustadistancia.edu.co

Carlos Felipe Hidalgo Reyes

Estudiante de Administración de Empresas, Universidad Santo Tomás modalidad distancia. Integrante Semillero de Investigación Estratega.

E-mail: carloshidalgo@ustadistancia.edu.co

Astrid Juliana Giraldo Caro

Estudiante de Administración de Empresas, Universidad Santo Tomás modalidad distancia. Integrante Semillero de Investigación Estratega.

E-mail: astridgiraldo@ustadistancia.edu.co
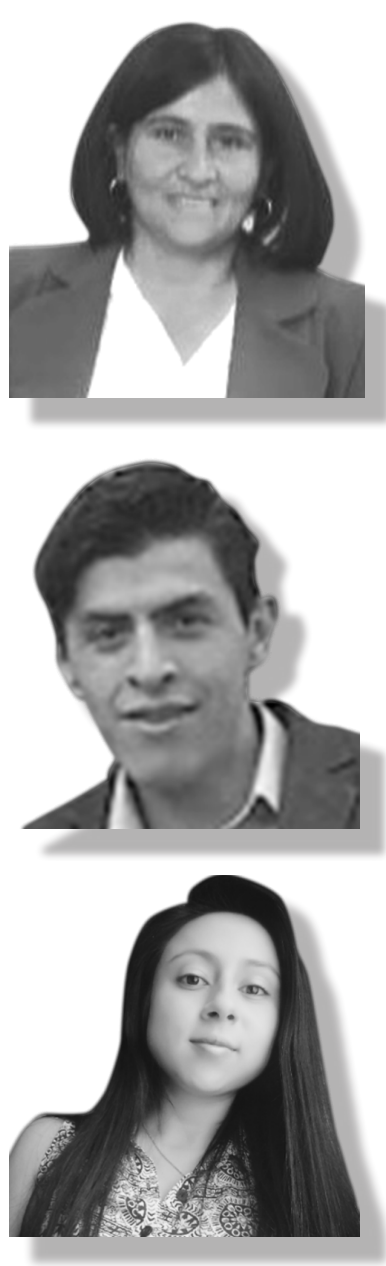


\section{RESUMEN}

A partir de la investigación denominada "Relación entre el mercado laboral y los egresados del Programa de Administración de Empresas de la VUAD (Vicerrectoría General de Universidad Abierta y a Distancia) en las ciudades de Villavicencio, Medellín y Bucaramanga", la cual se llevó a cabo en el año 2016; dando continuidad al trabajo similar desarrollado en el 2015 en la ciudad de Bogotá, donde los resultados generales coinciden en algunos aspectos, por ejemplo, que los empresarios de las diferentes ciudades objeto de estudio (Bucaramanga, Medellín y Villavicencio), consideran que dentro del perfil del administrador de empresas deben sobresalir las habilidades gerenciales, aquellas, que permiten al profesional tener la capacidad de trabajar bajo presión, proponer alternativas de solución a posibles problemas, contar con la capacidad para liderar equipos de trabajo, capacidad creativa y motivadora, entre otros aspectos que esperan los empresarios, el administrador de empresas tenga para ser un profesional competitivo.

En este orden de ideas, la investigación desarrollada durante el año 2016, pretendió conocer de cerca, al entrevistar y aplicar instrumentos de recolección de información (encuesta), la percepción que tienen tanto los empresarios como los egresados del programa de administración de empresas de la VuAD, frente al perfil con el cual son formados los estudiantes en su proceso académico, el cual busca fortalecer el conocimiento adquirido por medio del acercamiento a la realidad empresarial, elemento que es valorado de forma positiva por parte de los egresados y los empresarios con los cuales se tuvo la oportunidad de socializar.

El tema de las habilidades gerenciales toma fuerza luego de haber trabajado en el desarrollo de la investigación para la propuesta del presente artículo, gracias a los resultados donde se rescata por parte de empresarios y egresados, que el futuro administrador de empresas debe fortalecer sus habilidades de liderazgo, negociación, visión gerencial y empresarial, con el propósito de ser competitivo dentro de las organizaciones donde laboran o en su propia empresa.

Palabras Clave: egresado, habilidades, trabajo en equipo, liderazgo, motivación, TI.

\section{Abstract}

From the investigation named Relation between the labor market and the gone away ones from the Program of Administration of Companies of the VUAD in Villavicencio's cities, Medellin and Bucaramanga, which was carried out in the year 2016, giving continuity to the similar work developed in 2015 in the city of Bogota, where the general results coincide with some aspects, for example, that the businessmen of the different cities I object of study (Bucaramanga, Medellin and Villavicencio), think that inside the profile of the administrator of companies the managerial skills must stand out, those, which allow to the professional to have the aptitude to work under pressure, proposing alternatives of solution to possible problems, possessing(relying on) the aptitude to lead equipments.

In this order of ideas, the investigation developed during the year 2016, it tried to know closely, on having interviewed and to apply instruments of compilation of information, the perception that there have both the businessmen and the gone graduated ones from the program of administration of companies of the VUAD, opposite to the profile with which the students are trained in his academic process, which seeks to 
strengthen the knowledge acquired by means of the approximation to the managerial reality, element that is valued positively on the part of the gone graduated ones and the businessmen with which the opportunity to socialize was had

The topic of the managerial skills takes force after having been employed at the development of the investigation, for the offer of the present article, thanks to the results where there is rescued on the part of businessmen and graduated, that the future administrator of companies must strengthen his skills of leadership, negotiation, managerial and managerial vision, with the intention of being competitive inside the organizations where they work or in his own company.

Keywords: Graduate, management, teamwork, ability, leadership, TI.

\section{Introducción}

El haber participado en el desarrollado de dos investigaciones, donde se aborda la temática sobre relación entre el mercado laboral y el programa de administración de empresas de la VUAD en la ciudad de Bogotá, y la Relación entre el mercado laboral y los egresados del programa de Administración de Empresas de la VuAd en las ciudades de Villavicencio, Medellín y Bucaramanga, permite tener unas conclusiones claras frente a la percepción que tienen los empresarios y los mismos egresados de administración de empresas, acerca de los temas abordados en cada espacio académico visto durante el transcurso de la carrera profesional.

En el presente artículo se pretende rescatar una parte del resultado de la investigación denominada: Relación entre el mercado laboral y los egresados del programa de Administración de Empresas de la VUAD en las ciudades de Villavicencio, Medellín y Bucaramanga, donde al concluir la misma, se rescata el interés que tienen los empresarios por aquellos profesionales que sobresalen por sus habilidades gerenciales, las cuales deben involucrar: capacidad creativa, generación de ideas, liderazgo, trabajo en equipo, resolución de conflictos, entre otros aspectos que requieren los nuevos líderes de las organizaciones.

En este sentido, la Universidad Santo Tomás, en el programa de administración de empresas, modalidad distancia, tiene un reto muy grande, teniendo en cuenta que es el primer claustro a nivel universitario en Colombia y una de las primeras instituciones que fomenta la modalidad de formación a distancia, aportando de esta manera a incrementar el número de graduados de administración de empresas en Colombia, siempre con el compromiso de formar $y$ graduar profesionales con un perfil muy apropiado, según exigencias del mercado.

De acuerdo con lo anteriormente expuesto, es importante rescatar la cifra plasmada en el informe del Observatorio Laboral (2014), el cual indica, que entre el 2011 y el 2014, se titularon 21.195 universitarios del programa de Administración de Empresas (Observatorio Laboral, 2014), siendo una de las carreras con mayor demanda en Colombia, lo cual exige a instituciones de prestigio como la Universidad Santo Tomás, VuAD, formar profesionales idóneos, altamente competitivos, generadores de nuevasideas, con sentido de responsabilidad social y humanista, consciente de fortalecer día a día sus habilidades, aquellas que le permitirán abrirse paso en el mercado laboral.

\section{Antecedentes}

Caicedo Rolón (2011) y Lussier \& Achua (2016), precisan que el éxito de las profesiones en lo individual y el destino de las organizaciones 
están determinados por la eficienia del comportamiento de los líderes.

En este sentido desde la misión del programa de administración de empresas de la Universidad Santo Tomás-Vuad, hay un propósito claro frente a la calidad, así como sus objetivos, entre ellos, el que tiene como fin incentivar en el profesional una actitud crítica, creativa, dinámica e innovadora a través del conocimiento adquirido mediante la docencia, la investigación y la proyección social, que le permitan generar procesos de inserción en escenarios nacionales e internacionales.

Según (Castro Aguilera \& Marchant R., 2005) la organización en todo momento necesita gerentes que posean destrezas y habilidades que posibiliten a su equipo participar en un ambiente que estimula el compromiso y la participación en los distintos niveles de trabajo, favorezca el crecimiento para el aprendizaje, oriente al funcionario hacia los resultados y por ende al rendimiento individual y grupal de cada uno de ellos.

De acuerdo con (Covey, 2014), experto en management y liderazgo, quien habla acerca de los 7 hábitos de la gente altamente exitosa: ser proactivo, actuar teniendo una finalidad en mente, poner primero lo primero, pensar en ganar-ganar, se debe procurar primero entender y luego ser entendido, establecer sinergias y mejorar continuamente.

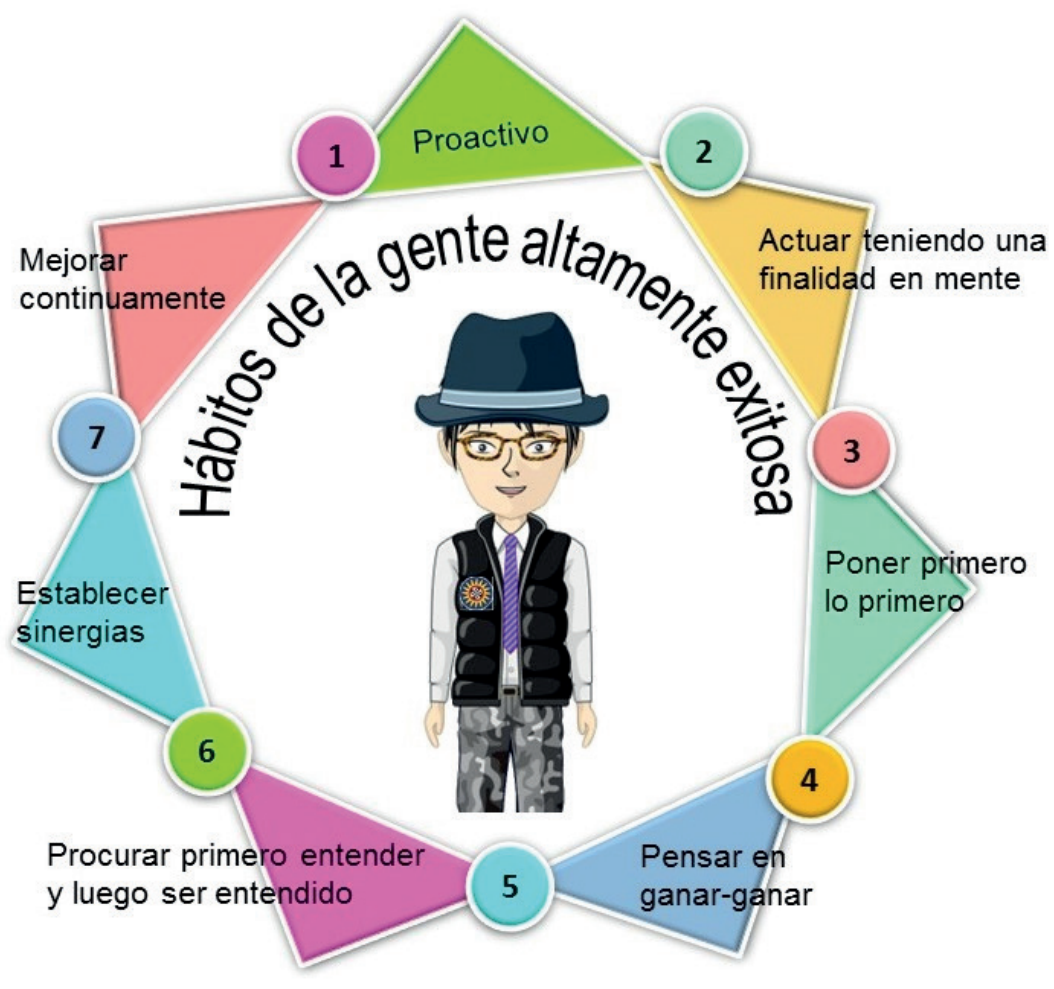

Figura 1. Hábitos de la gente altamente exitosa.

Resumen: lo expuesto por el autor Stephen Covey, frente a los hábitos que debe tener una persona exitosa, para el caso del presente artículo, para los gerentes altamente efectivos.

Fuente. Elaboración propia. 
Calderón Hernández \& Castaño Duque (2005) en su libro titulado: Investigación en administración en América Latina, exponen, una clasificación de habilidades gerenciales de la siguiente manera: negociación, liderazgo, pensamiento estratégico, manejo del conflicto, espíritu emprendedor, manejo del tiempo, toma de decisiones, comunicación y trabajo en equipo.

Partiendo de este pequeño análisis, el cual coincide con la información primaria recopilada en la investigación desarrollada, es evidente que las organizaciones actuales requieren de profesionales con mayor capacidad, por ejemplo, para llevar a cabo negociaciones, trabajo bajo presión, creatividad, liderazgo, pensamiento estratégico, así como una excelente disposición para el trabajo en equipo.

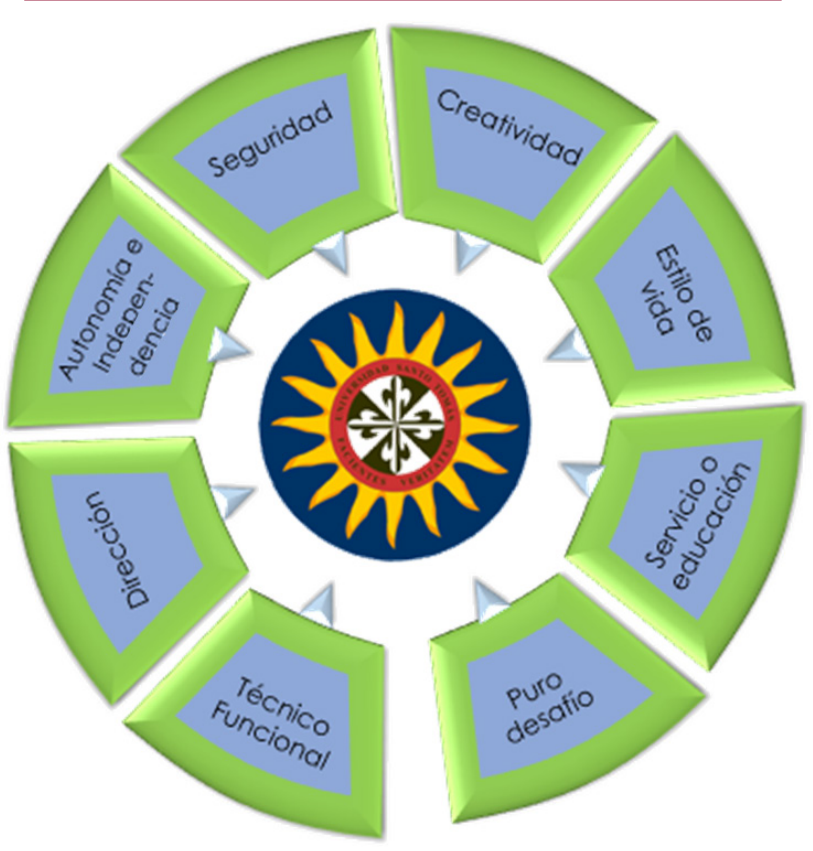

Figura 2. Hábitos de la gente altamente exitosa.

Resumen: gráfico sobre las anclas de la motivación planteadas en el modelo schein. Rescata elementos valiosos acerca de la visión que cada individuo puede tener según sus creencias y convicción a la hora de tomar una decisión, ejemplo, carrera profesional, ocupación, servicio, etc. Fuente. Elaboración propia.
Para Schein (2009), no todos los seres humanos son iguales, bilógicamente hablando, se nace con ciertas diferencias, que llevan a comportamientos distintos, es decir, lo que define el carácter de una persona y aptitudes o habilidades diferentes.

Cada persona a partir de sus características personales escoge su camino (en este caso, su carrera profesional) en torno a un "ancla" que es la que define y determina los objetivos de la persona.

Dichas anclas se clasifican en: autonomía e independencia, dirección, técnico funcional, creatividad, puro desafío, servicio o educación y estilo de vida.

La seguridad está asociada a la consecución de un trabajo seguro, ejemplo de muchos profesionales en Colombia, estabilidad, prestaciones sociales, y otros posibles beneficios.

Creatividad, muchos profesionales en diferentes ramas del conocimiento son muy creativos, aprovechando esta cualidad para explorar, crear, proponer y desarrollar sus propios emprendimientos, por ejemplo.

Autonomía e independencia, se busca tener libertad a la hora de llevar a cabo una carrera profesional, imprimiendo en cada acción su propio estilo, condición característica de muchos empresarios o microempresarios.

Dirección, en esta ancla se encuentran quienes desean dirigir una organización o coordinar equipos de trabajo, buscan retos a nivel gerencial, ejercer responsabilidades, tener participación dentro de la organización.

Técnico funcional, las personas se enfocan en especializarse en determinada área técnica, les gusta sentiré indispensables para la organización. 
Puro desafío, siempre en busca de grandes retos, cuando en la organización que están no pueden desarrollar retos, prefiere retirarse a buscar nuevos retos.

Servicio y dedicación, profesionales con un alto compromiso profesional, que dan un poco más porque piensan en aportar con su conocimiento y su parte humana.

Estilo de vida, personas que anteponen su vida familiar a la laboral, buscando en lo posible que su trabajo se adapte a sus necesidades(horarios, ubicación geográfica, condiciones de seguridad, educación, etc.), esto genera en el profesional un alto grado de motivación.

De acuerdo con el autor, se puede evidenciar con claridad que cada profesional, tiene unas características propias, las cuales contribuyen a que el profesional se destaque según su enfoque, siendo esencial el fortalecimiento de sus competencias, que le permitan ser más competitivo según su perfil y ocupación, trabajo que se lleva a cabo en la institución de educación superior, por medio del caso estudio, fortalecimiento teórico y desarrollo de trabajo práctico por parte del estudiante, quien identifica la problemática en determinadas áreas de una organización y a partir del diagnóstico, soportado con herramientas de diagnóstico, propone una serie de alternativas de solución.

\section{Metodología}

Para llevar a cabo el presente escrito, se contempló el trabajo desarrollado por los integrantes del Semillero de Investigación Estratega, en las dos investigaciones denominadas: "Relación entre el mercado laboral y los egresados del Programa de Administración de Empresas de la VUAD en la ciudad de Bogotá" y "Relación entre el mercado laboral y los egresados del Programa de Administración de Empresas de la VUAD en las ciudades de Villavicencio, Medellín y Bucaramanga”.

También se acude a la información trabajada por autores como: Koontz, informe Observatorio Laboral, Banco Mundial, Observatorio Laboral de Colombia, entre otras fuentes que abordan la realidad del profesional de administración de empresas y su perfil competitivo.

En cuanto a la consulta de autores, se tienen en cuenta: Madrigal (2009), Schein E. (2009), Arroyo Tovar (2017), Gutiérrez Tobar (2010), entre otros, quienes gracias a sus investigaciones han efectuado aportes frente al desarrollo y fortalecimento de las habilidades gerenciales.

\section{Resultados}

Tras el desarrollo de la investigación denominada "Relación entre el mercado laboral y los egresados del Programa de Administración de Empresas de la VuAD en las ciudades de Villavicencio, Medellín y Bucaramanga"yla constanteretroalimentación recibida en ponencias, tutorías, reuniones y opiniones del cuerpo docente y estudiantes de la universidad, se puede llegar a una conclusión muy importante, la cual se enfoca en que cada día surgen nuevos cambios, que exigen llevar a cabo estudios que permitan conocer de cerca lo que afecta o beneficia un proceso de formación, así como lo que el mercado laboral por ejemplo espera de los futuros profesionales.

De acuerdo con los requerimientos de personal en la organización, los cargos más ocupados por un administrador de empresas, corresponde a: 


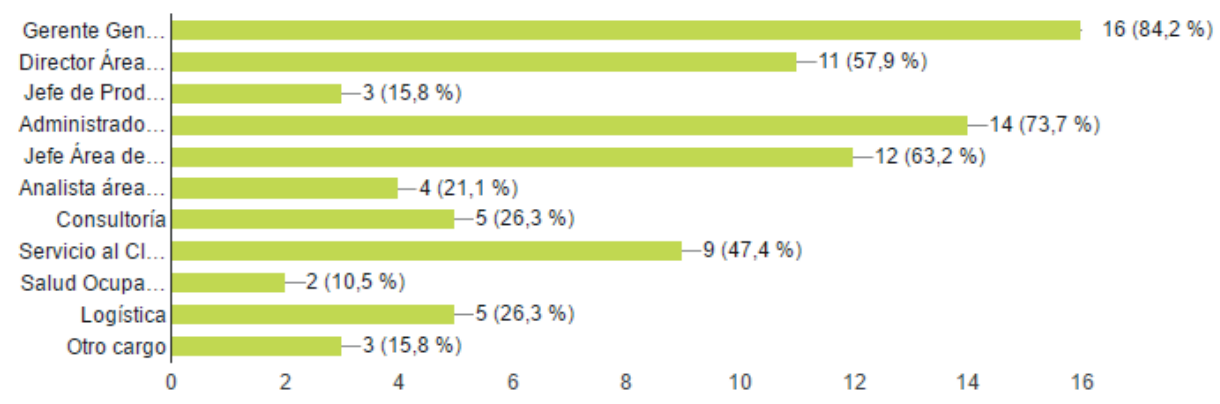

Gráfica 1. Resumen cargos más ocupados pro el administrador de empresas VuAD.

Resumen: La gráfica muestra según encuestas aplicadas, los diferentes cargos que ocupan los egresados del programa de administración de empresas de la VUAD.

Fuente. Elaboración propia.

Según los resultados del instrumento, los cargos más ocupados por el administrador de empresas, está en su orden: gerente general, director área comercial, administrador general, jefe área de talento humano, analista área financiera, servicio al cliente y logística.
Con relación a la percepción de valor que tienen los empresarios frente a las competencias genéricas de los egresados de pregrado administración de empresas, de la VUAD, se tiene:

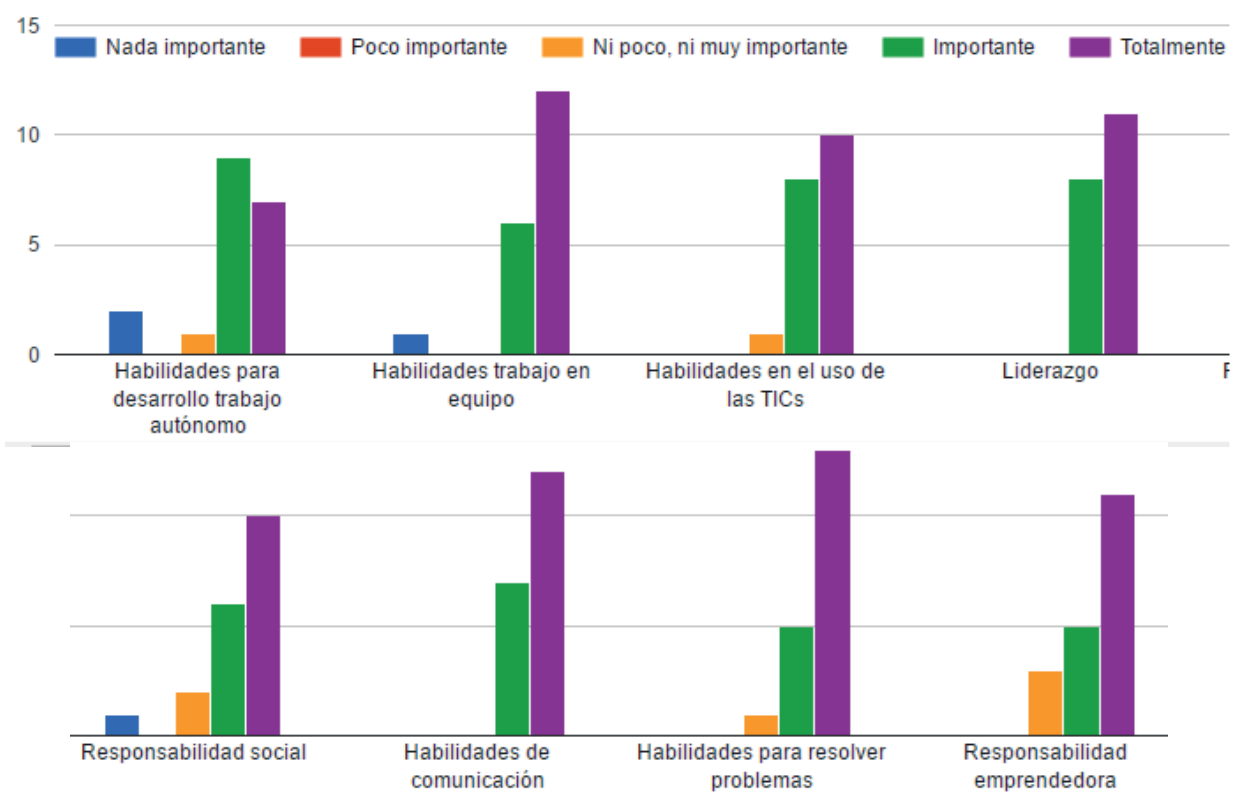

Gráfica 2. Resumen cargos más ocupados por el administrador de empresas VuAD.

Resumen: La gráfica muestra los resultados de la percepción de valor que tienen los empresarios frente a las competencias genéricas de los egresados de pregrado administración de empresas, de la VuAD.

Fuente. Elaboración propia. 
Frente a la percepción que tienen los empresarios sobre los egresados del programa de administración de empresas, se encuentra que es muy importante para los empresarios encuestados, que los candidatos cuenten con: habilidades de comunicación, las habilidades para resolver problemas, trabajo en equipo, responsabilidad emprendedora y el uso de las TIC.

Es de resaltar en este sentido, que cada día es más exigente el tema del fortalecimiento de las habilidades en los profesionales, porque las organizaciones van a un ritmo más acelerado, donde los cambios son constantes, lo que obliga a que los colaboradores de las organizaciones tengan que afrontar situaciones de cambio, retos tecnológicos, entre otros aspectos, que exige un alto nivel de profesionalismo por parte de cada miembro de la organización.

Para la organización es más conveniente que el profesional en el área de administración de empresas tenga mayor énfasis de formación, por ejemplo, en:

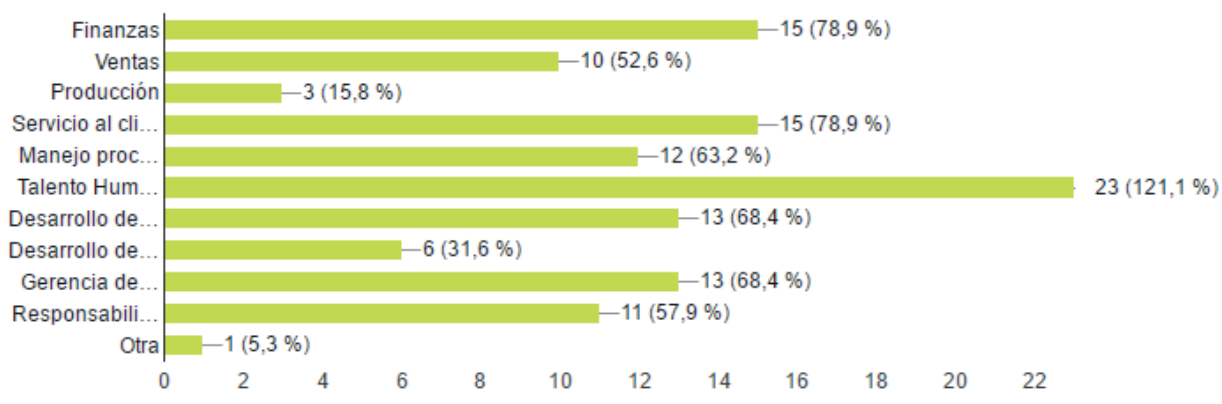

Gráfica 3. Resumen cargos más ocupados por el administrador de empresas VUAD.

Resumen: La gráfica muestra los resultados generales con relación a énfasis que según lo emprearios, debe tener un egresado de administración de empresas. Talento humano, finanzas, servicio al cliente, desarrollo de proyectos, gerencia de proyectos, manejo procesos TIC y responsabilidad social.

Fuente. Elaboración propia.

Lo anteriormente expuesto, resumen en gran medida las preguntas enfocadas al tema de habilidades gerenciales y que concuerdan con la información secundaria consultada frente a las expectativas que los empresarios pueden tener sobre los egresados del programa de administración de empresas.

La pregunta relacionada con el énfasis que debe tener un administrador de empresas permite tener una idea a la institución acerca de los énfasis que se deben fortalecer en el pregrado y los que se pueden trabajar a nivel de especialización, por ejemplo.
Partiendo del análisis anteriormente expuesto, donde se rescatan los puntos de vista de varios autores frente al tema de habilidades gerenciales, y la percepción de algunos empresarios y egresados del programa de administración de empresas de la VUAD, que participaron en el desarrollo de la investigación, donde los integrantes del Semillero de Investigación Estratega, tuvieron la oportunidad de trabajar, se rescata también la opinión de estos dos estudiantes, quienes no sólo diseñaron instrumentos, aplicaron encuestas, efectuaron entrevistas, construyeron informes y presentaron 
ponencias, sino que también tienen su punto de vista, el cual se plasma a continuación.

Carlos Felipe Hidalgo Reyes. Luego de 5 años de formación profesional, entre ellos 3 años de participación activa en el Semillero Estratega, se rescata el accionar del administrador de empresas, quien siempre está motivado por el deseo de aprender cada día más, por la perseverancia para el cumplimiento de sus metas, esfuerzo y dedicación, por adquirir competencias que contribuirán al desarrollo profesional en el escenario laboral. Todo esto visto como un conjunto de herramientas que en relación con los conocimientos brindados por la Universidad Santo Tomás, aportan elementos para fortalecer el sentido de liderazgo y abrir caminos exitosos; los cuales no solo dependen de una cátedra o tutoría, sino de una serie de acciones que de forma conjunta permiten abrirse un camino hacia un futuro empresarial, bien sea como colaborador de una organización o gerente de su propia empresa, donde las habilidades gerenciales cobrarán fuerza y serán el pilar que permitirá tener éxito.

La teoría adquiere verdadero valor cuando en el escenario de mercado laboral, se apropia el conocimiento y se lleva a la práctica, fortaleciendo día a día las habilidades gerenciales requeridas para hacer frente a la realidad de las organizaciones.

En el caso de uno de los estudiantes que participó en la investigación, se rescata el conocimiento de más de 10 años, entre la experiencia laboral y formación académica, la cual permite contar con las bases necesarias para crear su propia empresa, contribuyendo a la generación de empleo, colocando en práctica las habilidades técnicas, de conocimiento y humanas, aquellas, que permiten no sólo desarrollar empresa, sino que también contribuye para ser ejemplo a seguir por parte del equipo de trabajo.
Teniendo en cuenta los 3 años donde se hizo parte activa del Semillero de Investigación Estratega, se puede resaltar el esfuerzo que hace la universidad por convertir a personas que luchan por un sueño, ser profesional, en grandes conquistadores, gracias a la orientación, y acompañamiento en los procesos de formación. Así, como el interés y la perseverancia por adquirir conocimientos que contribuyen a fortalecer el sello tomasino en el futuro administrador de empresas. Es así que, como integrante del Semillero Estratega, se visualiza la relación que existe entre el programa de administración de empresas de la Universidad Santo Tomás-VUAD y el campo laboral con un 70/30; donde el 70\% es la disposición para adquirir y aplicar el conocimiento por parte del estudiante, el cual no se logra en un año, ni mucho menos en una práctica laboral, es un esfuerzo que día a día se debe lograr acompañado de ese 30\%, el cual se refleja en el fortalecimiento de las habilidades gerenciales, gracias a la experiencia que se gana en el sector real.

Astrid Juliana Giraldo. El iniciar una carrera profesional, trae consigo muchos retos y de igual manera oportunidades, en este caso, el de poder hacer parte de un semillero de investigación que permite alimentar tu conocimiento; conocer de cerca el proceso para desarrollar una investigación y el acercarse a la población objeto de estudio, para este caso, los estudiantes y egresados de la Universidad Santo Tomás, programa de administración de empresas, modalidad distancia, de las ciudades de Bogotá, Bucaramanga, Medellín y Villavicencio, donde se tuvo la oportunidad de viajar al CAU de Bucaramanga en la segunda fase de la investigación 2016.

Así mismo, se tuvo contacto con algunos de los empresarios, a quienes se les efectuaron algunas preguntas relacionadas con la percepción que ellos tienen frente a las competencias con las cuales un egresado de administración de empresas obtiene su 
título. Esto a partir de los temas abordados en cada espacio del programa que conforman el pensum académico.

Sin duda, el analizar los resultados tanto de la ciudad e Bogotá, como de las otras tres ciudades Bucaramanga, Medellín y Villavicencio, coincide en que el profesional en administración de empresas debe reunir una serie de cualidades que son fortalecidas con la experiencia , pero que implícito a su formación llevan un sello; el mismo que ha hecho grande a una institución, el sello tomasino, profesional humanista, solidario, creativo e innovador, comprometido con la sociedad, con el país, al ser un excelente profesional que gracias a sus capacidades y cualidades da lo mejor de sí a las organizaciones donde laboran, bien sea de un tercero o su propia empresa.

\section{Conclusiones}

Al concluir el presente artículo, el cual se enfocó en el tema de habilidades gerenciales, desde la visión del egresado, punto que se trató con algunos de los empresarios ya egresados que colaboraron con el desarrollo del instrumento aplicado, así como artículos consultados que rescatan la importancia del tema en los nuevos líderes empresariales y profesionales del área de administración, es posible rescatar, que la percepción de empresarios como egresados frente a los espacios académicos en los cuales se debe profundizar, están relacionados directamente con: creatividad e innovación, capacidad trabajo en equipo, técnicas de negociación, manejo de TIC, liderazgo, motivación, entre otros aspectos que contribuyen a formar los futuros profesionales, los cuales deben afrontar los diferentes retos que trae consigo cada organización según su modelo de negocio.

Una última conclusión y recomendación es que desde el claustro universitario se deben desarrollar estrategias encaminadas al acercamiento y seguimiento al egresado, de tal manera que él se sienta aún parte fundamental de la institución, que se sienta comprometido con la institución, que aproveche las diferentes actividades que se diseñan pensadas en ellos, esto, porque una de las barreras más grandes que tiene cualquier institución de educación superior para llevar a cabo un estudio con egresados, es el poder contactaros de forma satisfactoria y que ellos tengan la disposición para colaborar con los requerimientos del momento.

Investigar no es una tarea fácil para estudiantes de la modalidad distancia, pero tampoco un imposible, por el contario es un reto que vale la pena afrontar, porque permite fortalecer conocimiento y experiencias desde lo académico y profesional.

\section{REFERENCIAS}

Arroyo Tovar, R. (2017). Habilidades Gerenciales. Desarrollo dde destrezas, competencias y actitud. Bogotá D.C.: Ecoe Ediciones Ltda.

Caicedo Rolón, A. (junio de 2011). Necesidades de capacitación en habilidades. Respuestas. doi:https://doi.org/10.22463/ issn.0122-820X

Calderón Hernández, G., \& Castaño Duque, G. A. (2013). Investigación en administración en América Latina: evolución y resultados. Manizales: Ed. Universidad Nacional.

Castro Aguilera, F., \& Marchant R., L. (2005). Competencias (o Habilidades) Gerenciales: Una Herramienta Indispensable para el Desarrollo Organizacional. Actualizaciones para el Desarrollo Organizacional. 
Obtenido de http://www.eumed.net/libros-gratis/2005/lmr/7.htm

Colombia, O. L. (2014). Observatorio Laboral. Bogotá D.C.

Covey, S. (2014). Los 7 hábitos de la gente altamente efectiva. Paidos.

Gutiérrez Tobar, E. (2010). Competencias Gerenciales. Bogotá D.C.: Ecoe Ediciones Ltda.

Koontz, H., Weihrich, H., \& Cannice, M. (2012). Administración Una perspectiva global y empresarial. México: Mc Graw Hill.

Laboral, O. (2014). http://www.graduadoscolombia.edu.co/html/1732/w3-article-335501.html.

López, R. y. (2012). Dirección de Marketing. Fundamentos y aplicaciones. México DF: Alfaomega.

Lussier, R. N., \& Achua, C. F. (2016). Liderazgo. Teoría, aplicación y desarrollo de habilidades. México D.F.: Cengage Learning. Obtenido de https://issuu.com/cengagelatam/docs/liderazgo_lussier_issuu

Madrigal, B. (2009). Habilidades Gerenciales. México: McGrawHill.

R., C. a. (2005). Actualizaciones para el Desarrollo Organiacional. España: Eumed. Obtenido de https://goo.gl/5wpGZ6

Rolón, A. J. (2011). Necesidades de capacitación en habilidades gerenciales para los gerentes de producción del sector de la arcilla del área metropolitana de Cúcuta. Respuestas, 16(1), 30-37. Recuperado el 20 de 8 de 2017, de https://dialnet.unirioja. es/descarga/articulo/5364526.pdf
Sainz. (2016). Marketing. El Plan de Marketing en la práctica. España: Esic. Obtenido de https://books.google.es/ books?i$\mathrm{d}=$ Kh9AYSiVf64C\&printsec=copyrigh$\mathrm{t} \& \mathrm{hl}=\mathrm{es} \&$ source $=\mathrm{gbs} \_p u b \_i n f o \_r \# \mathrm{v}=0-$ nepage\&q\&f=false

Schein, E. (1980). Entrecodigos. Obtenido de Blog: http://www.entrecodigos. com/2009/o6/las-anclas-de-la-motivacion-de-edgar-schein.html

Schein, E. (2009). Cultura Organizacional y liderazgo. Sao Paulo: Atlas.

SEMANA, R. (Agosto de 1993). Revista Dinero. Obtenido de http://www.semana. com/nacion/articulo/historia-triunfador/20343-3

Stephen, C. (2012). Los siete hábitos de la gente altamente exitosa. 2. Obtenido de http:// clavesliderazgoresponsable.blogspot.com. co/2012/07/stephen-covey-los-siete-habitos-de-la.html 